\title{
Universiteit
}

Leiden

The Netherlands

\section{Membrane sweeping and prevention of post-term pregnancy in low- risk pregnancies: a randomised controlled trial}

Miranda, E. de; Bom, J.G. van der; Bonsel, G.J.; Bleker, O.P.; Rosendaal, F.R.

\section{Citation}

Miranda, E. de, Bom, J. G. van der, Bonsel, G. J., Bleker, O. P., \& Rosendaal, F. R. (2006). Membrane sweeping and prevention of post-term pregnancy in low-risk pregnancies: a randomised controlled trial. Bjog - An International Journal Of Obstetrics And Gynaecology, 113(4), 402-408. Retrieved from https://hdl.handle.net/1887/5014

Version: $\quad$ Not Applicable (or Unknown)

License:

Downloaded from: https://hdl.handle.net/1887/5014

Note: To cite this publication please use the final published version (if applicable). 


\title{
Membrane sweeping and prevention of post-term pregnancy in low-risk pregnancies: a randomised controlled trial
}

\author{
E de Miranda, ${ }^{a}$ JG van der Bom, ${ }^{b}$ GJ Bonsel, ${ }^{c}$ OP Bleker, ${ }^{a}$ FR Rosendaal ${ }^{b}$ \\ ${ }^{a}$ Academic Medical Centre, Department of Obstetrics and Gynaecology H4-210, Amsterdam, The Netherlands beiden University Medical Centre, \\ Department of Clinical Epidemiology C9-P, Leiden, The Netherlands ${ }^{c}$ Academic Medical Centre, Department of Social Medicine-Public Health \\ Epidemiology J3-313, Amsterdam, The Netherlands \\ Correspondence: E de Miranda, Academic Medical Center, Department of Obstetrics and Gynaecology H4-210, PO Box 22700, 1100 DE Amsterdam, \\ The Netherlands. Email est.demiranda@inter.NL.net
}

Accepted 4 January 2006. Published OnlineEarly 20 February 2006.

Objective To evaluate the effectiveness of membrane sweeping at 41 weeks for the prevention of post-term pregnancy.

Design A multicentre randomised controlled trial.

Setting Fifty-one primary care midwifery practices in the Netherlands.

Population A total of 742 low-risk pregnant women at 41 weeks of gestation.

Methods Participants were randomly assigned to serial sweeping of the membranes (every 48 hours until labour commenced up to 42 weeks of gestation) or no intervention.

Main outcome measures Post-term pregnancy ( $\geq 42$ weeks). Subgroup analyses were performed on nulliparous and parous women. Secondary outcomes included adverse effects.

Results Serial sweeping of the membranes at 41 weeks decreased the risk of post-term pregnancy (87/375 [23\%] versus $149 / 367$
[41\%]; relative risk [RR] 0.57, 95\% CI 0.46-0.71; number needed to treat [NNT] 6 [95\% CI 4-9]). Benefits were also seen in both subgroups (nulliparous: 57/198 [29\%] versus 89/192 [46\%]; RR 0.62 [95\% CI 0.48-0.81]; NNT 6 [95\% CI 4-12] and parous: 30/ 177 [17\%] versus 60/175 [34\%]; RR 0.49 [95\% CI 0.34-0.73]; NNT 6 [95\% CI 4-6]). Adverse effects were similar in both the groups except for uncomplicated bleeding, which was reported more frequently in the sweeping group. Other obstetric outcomes and indicators of neonatal morbidity were similar in both groups. There were two perinatal deaths in each group.

Conclusions Membrane sweeping at 41 weeks can substantially reduce the proportion of women with post-term pregnancy.

Keywords Induction of labour, onset of labour, membrane sweeping, post-term pregnancy.

Please cite this paper as: de Miranda E, van der Bom J, Bonsel G, Bleker O, Rosendaal F. Membrane sweeping and prevention of post-term pregnancy in low-risk pregnancies: a randomised controlled trial. BJOG 2006; 113:402-408.

\section{Introduction}

Post-term pregnancy (gestational age of $\geq 294$ days $[\geq 42$ weeks $]^{1}$ ) is associated with increased perinatal morbidity and mortality. ${ }^{2}$ The incidence of post-term pregnancy ranges from 4 to $18 \%,{ }^{3}$ depending on the method of determination of the gestational age, the subject population and the local practice patterns. Sweeping the membranes (digital separation of the membranes from the lower uterine segment) is an old and simple method ${ }^{4}$ to promote spontaneous onset of labour, which is regularly applied to prevent post-term pregnancy, although its effectiveness in relation to the optimal timing of the procedure is still unclear. Membrane sweeping causes an increase in prostaglandin metabolites in the maternal circulation and in local prostaglandin production., ${ }^{5,6}$ Both are associated with ripening of the cervix and, ultimately, with spontaneous onset of labour.

The results of trials on the effectiveness of membrane sweeping have been inconsistent, ${ }^{7-25}$ possibly due to methodological differences between studies. ${ }^{7}$ Routine use of membrane sweeping between 38 and 40 weeks does not seem to produce clinically important benefits according to a recent Cochrane review; ${ }^{7}$ yet, it might be beneficial in women with a gestational age of 41 weeks. ${ }^{15,16}$ Our aim was to assess the effectiveness of membrane sweeping starting at 41 weeks for 
the prevention of post-term pregnancy among a low-risk population in a primary care setting.

\section{Methods}

A multicentre randomised trial was conducted in 51 midwifery practices throughout the Netherlands between June 2000 and March 2003. Low-risk pregnant women were eligible for inclusion in the trial when they were low risk (single fetus in cephalic presentation, no pregnancy complications or risk factors and no contraindications to normal vaginal delivery), with a reliable gestational age of 41 weeks (range $40^{+6}$ to $41^{+3}$ ) and no history of blood loss after the first trimester or suspicion of loss of amniotic fluid during pregnancy. The primary outcome was post-term pregnancy, which was defined as a gestational age of 294 days or more. A referral to the local obstetrician for surveillance or induction of labour was programmed at 42 weeks. Induction of labour was scheduled by the obstetricians according to local hospital protocols and varied from induction at $42^{+0}$ to expectant management until $43^{+0}$ weeks. For this reason, formal induction of labour was not suitable as a primary outcome measure.

At 39 weeks of gestation, all the eligible women received a written information about the trial, and at 40 weeks, they were invited to participate. A written informed consent was obtained at the antenatal visit of 41 weeks, after which the participating woman opened the next successive randomisation envelope.

Randomisation in this open trial was accomplished by blocked randomisation using 30 blocks of $25,{ }^{26}$ with a variable allocation ratio of 12:13 or 13:12. The allocations were placed within consecutively numbered, opaque, sealed envelopes. A box containing the agreed number of randomisations (variable for each centre) was then sent to the midwifery practices where they were kept. The participating midwives were unaware of the randomisation method. Stratification by centre was performed in order to reveal any differences according to midwifery practice.

After every randomisation, the numbered envelope containing the allocation card was posted to the trial coordinator together with a randomisation form containing the date of randomisation, the allocation group and the subject characteristics.

Women allocated to the control group received routine monitoring. To prevent prostaglandin release, vaginal examination was not performed in the control group until the onset of labour. In addition, we asked the midwives to refrain from advice regarding sexual intercourse as a way of stimulating labour onset, regardless of the allocation. Women allocated to sweeping received routine monitoring as well, followed by a vaginal examination for assessment of the cervical ripeness (Bishop score $[\mathrm{BS}])^{27}$ and immediate sweeping. Sweeping was performed by separating the lower membranes as much as possible from their cervical attachment, with three circumferential passes of the examining fingers. When sweeping was not possible because the cervix was closed, cervical massage was performed. ${ }^{15}$ Massage of the cervical surface was performed with circular pushing and massaging movements of the fore finger and middle finger for approximately 15 seconds. Sweeping was repeated every 48 hours, with a maximum of three times, until labour commenced or 42 weeks of gestation was reached. The midwives explained to the women who had been swept that blood-stained mucus or painful contractions could occur.

The ethics committee of the Academic Medical Center of Amsterdam approved the trial.

Data concerning prenatal care, obstetric intervention, delivery and infant condition were recorded on a case report form (CRF). We also collected data on the adverse effects and the woman's satisfaction by self-reported questionnaires. If labour did not start within 48 hours, a questionnaire assessing possible adverse effects such as contractions, nature of the contractions and vaginal bleeding was completed. The midwives asked all women to complete the questionnaires.

The primary endpoint of the trial was delivery at or beyond 42 weeks. The sample size was calculated based on estimations contained in previous reports on the future of Dutch obstetric practice $^{28}$ and based on data of the Perinatal Database of the Netherlands (LVR). ${ }^{29}$ Both the reports are based on detailed data regarding pregnancy, birth and infant condition from 95\% of Dutch midwives and obstetricians. For an expected difference favouring sweeping of $10 \%$, i.e. $30 \%$ instead of $40 \%$ post-term deliveries, with an alpha of 0.05 and a beta of 0.20 , two groups of 375 women were required. We computed relative risks (RR) to compare crude and stratified proportions and calculated the 'number needed to treat (NNT)' with 95\% confidence limits. Kaplan-Meier analysis was used to describe postponement ('survival') from randomisation to postterm pregnancy, and additional logistic regression analysis was performed to adjust the comparison of proportions for centre effects. Data analysis was performed using SPSS software (SPSS, Chicago, IL, USA).

\section{Results}

From June 2000 to March 2003, 141 midwives from 51 midwifery practices randomised 750 women. Allocation was balanced (difference $\leq 2$ ) within 44 practices and unbalanced (difference 3-6) in 7 practices. Eight women were excluded from the analysis because they did not meet the inclusion criteria (five controls, one sweeping) or were lost to follow up (one in each group; Figure 1). We included two women allocated to control and one woman allocated to sweeping who were unintentionally randomised at a gestational age of $40^{+5}$ and one woman allocated to sweeping who was randomised at a gestational age of $41^{+5}$. 


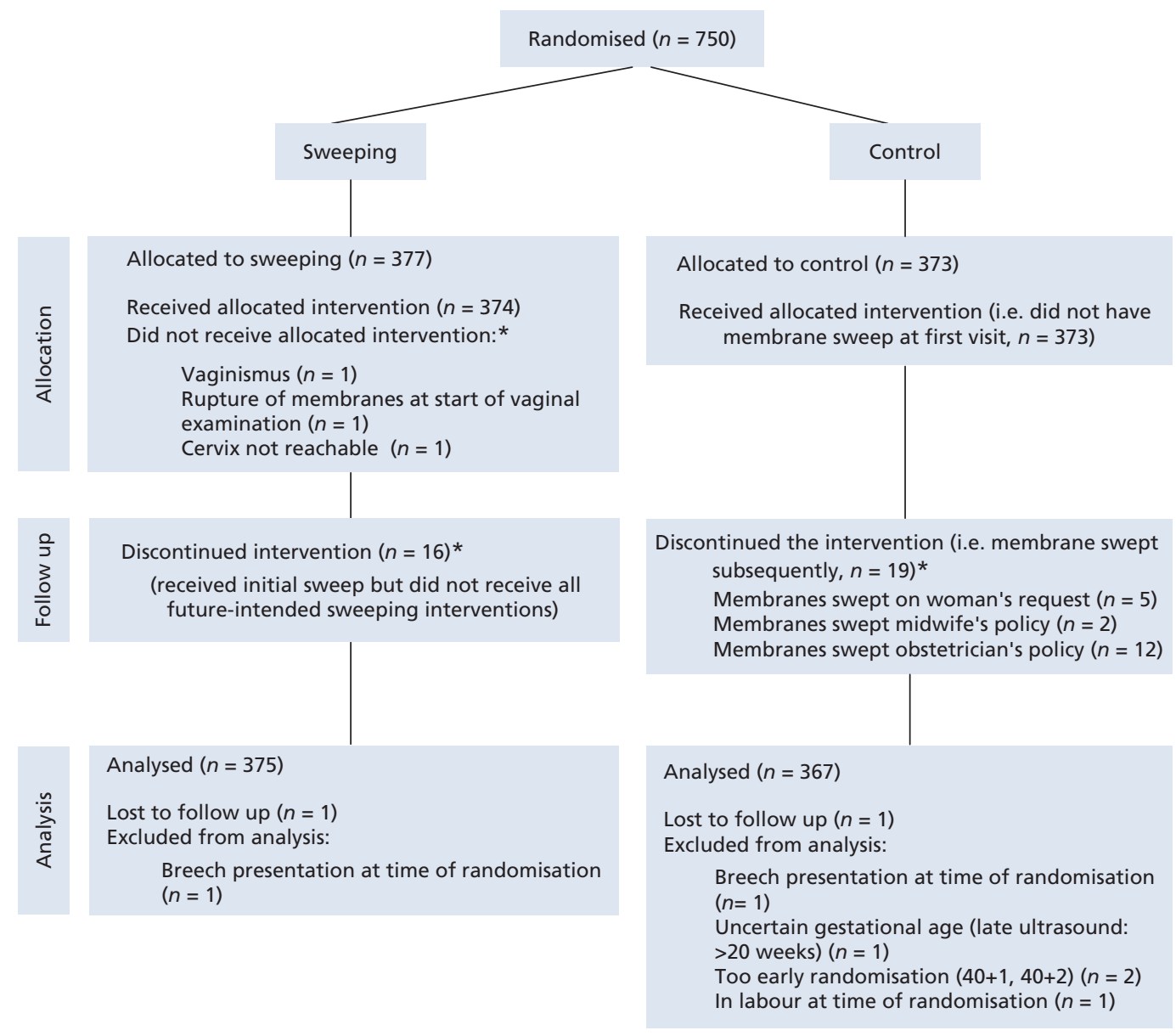

Figure 1. Flow diagram of participants through each stage of the sweeping trial.*Included in the analysis under 'intention to treat'.

Primary analysis was by intention to treat, i.e. three women allocated to sweeping, who did not receive the intervention, and 19 women randomised to the control group, who were nevertheless swept, were analysed according to the allocated group. This left 742 women to be analysed, 375 in the sweeping group and 367 in the control group (Figure 1).

Questionnaires from the participants were available in 687 cases (93\%). The CRFs of 22 women allocated to control and 11 women allocated to sweeping were lost, mostly during hospitalisation. Data on the main outcomes for these 33 women could be collected in all cases from the midwifery dossiers and the hospital files, but information on BS, adverse effects and subject's satisfaction was missing.

The baseline characteristics of the groups were similar (Table 1). Both the groups contained slightly more nulliparous women than parous women. The median BS at baseline in the sweeping group was 4 (inter quartile range [IQR] 2-5). BS of nulliparous and parous women were similar at baseline (median BS among nulliparous women: 4 [IQR 2-5], and among parous women: 4 as well [IQR 3-5]). There were 283 women with a BS of $<6$ at baseline and 81 women with a BS of $\geq 6$. Gestational age was determined by ultrasound before 18 weeks in 595 women $(80 \%)$ or by certain last menstrual period corresponding with initial examination in 147 women $(20 \%)$.

Table 1. Characteristics of study participants, according to group

\begin{tabular}{|c|c|c|c|c|}
\hline & \multicolumn{2}{|c|}{$\begin{array}{l}\text { Sweeping } \\
(n=375)\end{array}$} & \multicolumn{2}{|c|}{$\begin{array}{l}\text { Control } \\
(n=367)\end{array}$} \\
\hline & Median & IQR & Median & IQR \\
\hline $\begin{array}{l}\text { Maternal age } \\
\text { (years) }\end{array}$ & 31 & $28-33$ & 31 & $28-34$ \\
\hline $\begin{array}{l}\text { Gestational } \\
\text { age (days) } \\
\text { at recruitment }\end{array}$ & 288 & $287-289$ & 288 & $287-289$ \\
\hline \multicolumn{5}{|l|}{ Parity } \\
\hline Nulliparous & $198(53)$ & & $192(52)$ & \\
\hline Multiparous & $177(47)$ & & $175(48)$ & \\
\hline
\end{tabular}

Values are given as median, IQR or numbers (\%). 
Sweeping significantly reduced the proportion of post-term pregnancies, which occurred in $23 \%$ of the women allocated to sweeping and in $41 \%$ of the controls (Table 2). The effect was observed both in nulliparous and parous women. Adjustment for centre revealed no difference with the crude estimate (results not shown). When the analysis was restricted to women who had a first trimester ultrasound, the effect on post-term pregnancy was similar: 66/299 (22\%) versus
121/296 (41\%), RR 0.54 (95\% CI 0.42-0.70). Re-analysis with all the excluded women included did not affect the overall RR.

In the intervention group, 76 of 283 (27\%) women with a BS of $<6$ at baseline and 7 of $81(9 \%)$ women with a BS of $\geq 6$ had a post-term pregnancy. Of the 375 women allocated to sweeping, 103 received cervix massage initially because of the impossibility of sweeping (nulliparous 67 and parous 36) and 65 women had massage of the cervix at all examinations. Of these 65 women,

Table 2. Outcomes according to sweeping or control

\begin{tabular}{|c|c|c|c|c|}
\hline & $\begin{array}{l}\text { Sweeping } \\
(n=375)\end{array}$ & $\begin{array}{l}\text { Control } \\
(n=367)\end{array}$ & $\begin{array}{l}\text { RR } \\
(95 \% \mathrm{Cl})\end{array}$ & $\begin{array}{l}\text { NNT } \\
(95 \% \mathrm{Cl})\end{array}$ \\
\hline \multicolumn{5}{|l|}{ Labour onset } \\
\hline Post-term pregnancy & $87(23)$ & $149(41)$ & $0.57(0.46-0.71)$ & $6(4-9)$ \\
\hline Nulliparous & $57 / 198(29)$ & $89 / 192(46)$ & $0.62(0.48-0.81)$ & $6(4-12)$ \\
\hline Multiparous & $30 / 177(17)$ & $60 / 175(34)$ & $0.49(0.34-0.73)$ & $6(4-12)$ \\
\hline Spontaneous onset of labour $<42$ weeks & $253(68)$ & $198(54)$ & $1.25(1.11-1.41)$ & \\
\hline Spontaneous onset of labour $\geq 42$ weeks & $32(9)$ & $53(14)$ & $0.59(0.39-0.89)$ & \\
\hline Prelabour caesarean section $<42$ weeks* & 0 & 1 & & \\
\hline Labour induction & $90(24)$ & $115(31)$ & $0.77(0.61-0.97)$ & \\
\hline$<42$ weeks & $35(9)$ & $19(5)$ & $1.80(1.06-3.08)$ & \\
\hline Impending post-term pregnancy & 8 & 4 & & \\
\hline 24 hours rupture of membranes & 11 & 4 & & \\
\hline On request & 4 & 1 & & \\
\hline Other** & 12 & 10 & & \\
\hline$\geq 42$ weeks & $55(15)$ & $96(26)$ & $0.56(0.42-0.75)$ & \\
\hline Post-term pregnancy & 51 & 92 & & \\
\hline$>24$ hours rupture of membranes & 2 & 1 & & \\
\hline Other & 2 & 3 & & \\
\hline \multicolumn{5}{|l|}{ Mode of labour induction } \\
\hline Oxytocin only & $51(14)$ & $56(15)$ & $0.89(0.63-1.26)$ & \\
\hline Started with prostaglandins & $33(9)$ & $51(14)$ & $0.63(0.42-0.96)$ & \\
\hline $\begin{array}{l}\text { Started with artificial rupture of membranes } \\
\text { (performed by the midwife) }\end{array}$ & $6(2)$ & $8(2)$ & $0.73(0.27-2.01)$ & \\
\hline Prelabour rupture of membranes*** & $57(19)$ & $50(19)$ & $1.03(0.73-1.44)$ & \\
\hline$>24$ hours ruptured membranes & $16(4)$ & $12(3)$ & $1.31(0.63-2.72)$ & \\
\hline Augmentation of labour & $47(13)$ & $40(11)$ & $1.15(0.76-1.75)$ & \\
\hline False labour & $21(6)$ & $15(4)$ & $1.37(0.72-2.62)$ & \\
\hline Fever during labour & $7(2)$ & $4(1)$ & $1.71(0.51-5.80)$ & \\
\hline Fever $\left(\leq 38^{\circ} \mathrm{C}\right)$ & 7 & 3 & & \\
\hline Fever $\left(>38^{\circ} \mathrm{C}\right)$ & 0 & 1 & & \\
\hline Meconium-stained amniotic fluid & $88(24)$ & $87(24)$ & $0.99(0.76-1.28)$ & \\
\hline \multicolumn{5}{|c|}{ Analgesia during labour (not for caesarean section) } \\
\hline Epidural & $17(5)$ & $14(4)$ & $1.19(0.60-2.38)$ & \\
\hline Pethidine & $47(13)$ & $45(12)$ & $1.02(0.70-1.50)$ & \\
\hline \multicolumn{5}{|l|}{ Mode of delivery } \\
\hline Spontaneous & $283(76)$ & $279(76)$ & $0.99(0.92-1.08)$ & \\
\hline Forceps & $6(2)$ & $4(1)$ & $1.47(0.42-5.16)$ & \\
\hline Vacuum & $49(13)$ & $49(13)$ & $0.98(0.68-1.42)$ & \\
\hline Caesarean section & $37(10)$ & $35(10)$ & $1.04(0.67-1.61)$ & \\
\hline Adverse neonatal outcomes & $30(8)$ & $29(8)$ & $1.01(0.60-1.70)$ & \\
\hline
\end{tabular}


$34(52 \%)$ had a post-term pregnancy compared with 30/242 (12\%) in the sweeping-only group (RR 4.22 [95\% CI 2.836.16]). In the control group, 19 women were swept, mainly after referral because of impending post-term pregnancy. Of these 19 women, 13 continued to post-term pregnancy.

Sweeping reduced the time between randomisation and delivery by 1 day (3.50 versus 4.47 days, mean difference 0.97 days, 95\% CI 0.60-1.35). Survival curves describing the cumulative probability of delivery before 42 weeks are shown in Figure 2. Sweeping significantly increased spontaneous onset of labour before 42 weeks (Table 2), mainly during the first 2 days (data not shown). Induction of labour before 42 weeks was also significantly increased in the sweeping group, mainly as a consequence of labour induction for $>24$ hours rupture of membranes (Table 2). The need for labour induction $\geq 42$ weeks was significantly decreased in the sweeping group. The positive effect of sweeping on spontaneous onset of labour was seen in nulliparous as well as in parous women. Sweeping significantly increased the likelihood of delivery in a primary care setting (188/375 versus $150 / 367$, RR 1.23 [95\% CI 1.05-1.44]), but analysis according to parity showed that the significant effect was restricted to parous women (nulliparous 69/198 versus 61/192, RR 1.10 [95\% CI $0.83-1.45]$ and parous $119 / 177$ versus $89 / 175$, RR 1.32 [95\% CI 1.11-1.58]).

Other obstetric and neonatal outcomes are summarised in Table 2. Labour induction with prostaglandins was reduced in the sweeping group. When stratified according to parity, sweeping only reduced the incidence of labour induction

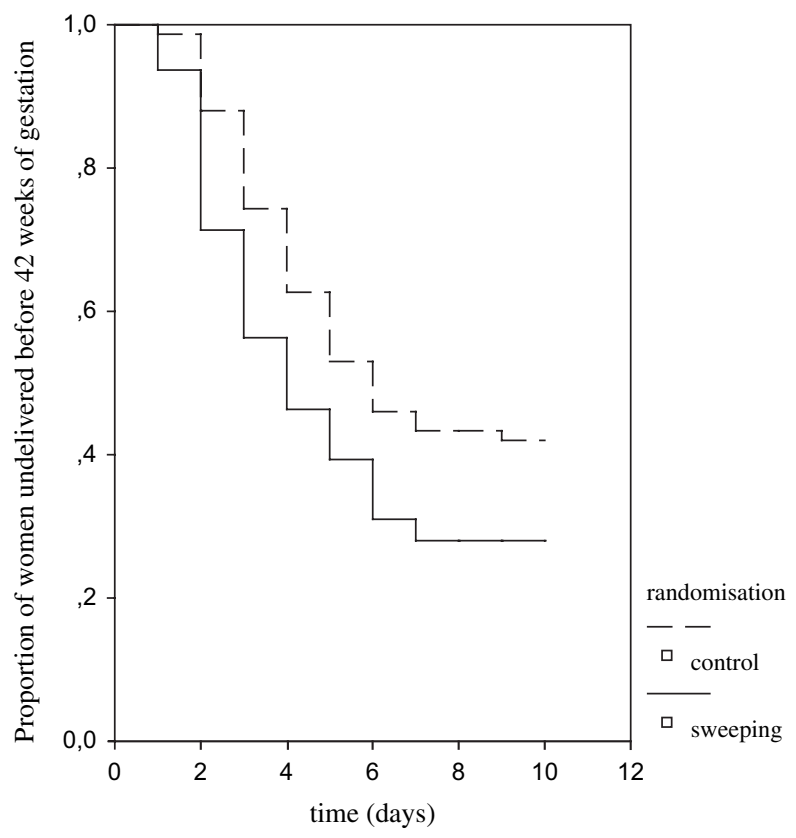

Figure 2. Survival curve for women between randomisation and 42 weeks. among parous women (27/177 versus 47/175, RR 0.57 [95\% CI $0.37-0.86])$, with no effect in nulliparous women $(57 / 198$ versus 60/192, RR 0.92 [95\% CI 0.68-1.25]). There were no differences in other obstetric outcomes such as rupture of membranes before onset of labour, $>24$ hours ruptured membranes, augmentation of labour, false labour, fever during labour, analgesia during labour and mode of delivery. Adverse neonatal outcomes were similarly frequent in both groups (Table 2), with no difference in Apgar score $<7$ at 5 minutes or admission to the neonatal care unit (or in the indications for admission there). There were four perinatal deaths, two in each group. In the sweeping group, one fetal death occurred at a gestational age of $41^{+6}$; the umbilical cord was looped around the baby's neck six times. The second perinatal death occurred 36 hours after an uncomplicated term delivery $\left(41^{+3}\right)$. A respiratory arrest took place 33 hours after delivery, resuscitation failed and the infant died 3 hours later. Post-mortem and bacterial cultures revealed that the probable course of death was group B streptococcal disease (GBS). In the control group, there was one unexplained death at 42 weeks after a failed vacuum extraction, followed by caesarean section, and one perinatal death because of lung and kidney hypoplasia.

Adverse effects reported until 48 hours after randomisation were similar in both the groups, except for bleeding, which was reported more frequently in the sweeping group (111/364 versus 16/345, RR 6.58 [95\% CI 3.98-10.87]). The frequency and character of contractions before onset of labour was similar in both the groups, but the duration of the contractions tended to be longer in the sweeping group (data not shown). Membrane sweeping was 'not painful' according to 111 women (31\%). However, 179 (51\%) women judged sweeping to be 'somewhat painful', while 60 (17\%) experienced sweeping as 'painful' or 'very painful'. In no instance did the procedure have to be stopped because of pain. After delivery, $88 \%$ (312/353) indicated that they would choose membrane sweeping in a next pregnancy. Even among the 239 women who described sweeping as painful, 210 (88\%) reported that they would choose membrane sweeping again in the next pregnancy.

\section{Discussion}

We performed a randomised trial to compare the effects of sweeping, with routine monitoring among low-risk pregnant women at a gestational age of 41 weeks. Membrane sweeping substantially reduced the number of post-term pregnancies and increased spontaneous onset of labour before 42 weeks.

Our study design tried to build on problems that are discussed in the Cochrane review on sweeping and on suggestions for future study made there and in previous trials. A major limitation of the systematic review concerned the relatively small sizes of the included studies; a large-scale trial 
on membrane sweeping was lacking. Because efficacy was expected to be low at an earlier gestational age and because the major concern is delivery beyond 42 weeks, we started the intervention at 41 weeks. In addition, to avoid interference with obstetric indications for induction of labour before 42 weeks, we evaluated sweeping in a low-risk population in a primary care setting. A major difference with most trials, in which sweeping was performed by one or two obstetricians, was the participation of many different midwives, ${ }^{30}$ implying that our results reflect real practice. We also followed the suggestion of a strategy of multiple successive sweeping ${ }^{10,18}$ rather than a single intervention.

Two characteristics of our trial merit discussion. First, we contrasted a strategy of serial sweeping to no sweeping. Our design does not, therefore, permit any conclusion as to whether serial sweeping is superior to single sweeping. Second, we did not determine BS in the control group, to avoid an effect of this procedure. Given the size of the groups and the randomisation process, it is unlikely that the initial BS differed between the two groups. Since we did not measure BS in the control group, it was not possible to show the effect of sweeping or massage on the ripening of the cervix, or the effect of sweeping for various BS. Indirectly, the effect from sweeping on the ripening of the cervix can be inferred from the reduced need for prostaglandins for induction of labour in the intervention group. At baseline, BS, as determined in the group randomised to sweeping, were low and not different between parous and nulliparous women, which supports the observations of Harris et al. ${ }^{31}$

It has been argued on theoretical grounds that sweeping should be more beneficial in parous women. Previous trials, however, did not confirm this. Although in our trial, the RR reduction was larger in parous women than in nulliparous women, sweeping was effective in both groups and the absolute risk difference (NNT) was the same. Nevertheless, a substantial positive effect of sweeping on the occurrence of 'spontaneous onset of labour before 42 weeks and spontaneous vaginal delivery' and 'delivery in a primary care setting' could only be observed for parous women. Furthermore, although sweeping reduced the overall incidence of labour induction, this effect was also only seen in parous women. These outcomes, however, relate to subgroup analyses, and the power of these to detect real but small differences is low.

Sweeping reduced the time between randomisation and delivery by 1 day. This shift in time is reflected in the occurrence of spontaneous onset of labour and of labour induction in both groups. Spontaneous onset of labour before 42 weeks was increased in the sweeping group, while spontaneous onset of labour $\geq 42$ weeks was increased in the control group. Labour induction before 42 weeks, on the other hand, was increased in the sweeping group, while induction $\geq 42$ weeks was increased in the control group. Women in both groups had labour induction $<42$ weeks on request or because of impending post-term pregnancy. For logistical reasons (office closure over the weekend), referral to the obstetrician occurred in some occasions 1 or 2 days before 42 weeks of gestation. The increase seen in labour induction before 42 weeks in the intervention group was partly due to an increase in $>24$ hours rupture of membranes. However, there was no difference seen in the total frequency of $>24$ hours rupture of membranes between the groups. Some previous trials have raised a concern about an increase in prelabour rupture of membranes with sweeping. ${ }^{10,16}$ Although one accidental rupture of membranes occurred at the start of the sweeping procedure, we observed no difference in the frequency of prelabour rupture of membranes between the sweeping group and the control group, which is in agreement with most other trials on sweeping. ${ }^{7,9,11,12,17,25}$

An important limitation of randomised trials such as ours is that they are seldom large enough to study rare adverse effects. In previous studies, no harmful adverse effects of sweeping were reported. ${ }^{7}$ In the study of Allott and Palmer ${ }^{8}$, there was one case of GBS in the control group. In our study, one perinatal death, probably because of early onset of GBS disease, occurred in the sweeping group. Thus far, membrane sweeping has not been associated with GBS. ${ }^{32-36}$ Consequently, the revised guidelines from Centers for Disease Controls and Prevention for the prevention of perinatal GBS did not recommend avoiding of membrane sweeping in GBScolonised women. ${ }^{37}$ However, as this disease occurs so rarely, a relation with sweeping is difficult to establish. Future studies, preferably case-control studies, need to address the effect of sweeping on perinatal GBS disease.

In our study, $17 \%$ of the women experienced sweeping as painful, which is roughly the same as reported previously, ${ }^{30}$ when $22 \%$ of women experienced the procedure as painful. In concordance with these results, women allocated to sweeping had a positive judgement on the intervention.

\section{Conclusions}

Even assuming the lowest incidence of post-term pregnancy of $4 \%$, membrane sweeping at 41 weeks will substantially reduce the proportion of women with post-term pregnancy. It is a simple and effective method that can be applied in out of hospital settings worldwide.

\section{Acknowledgements}

A special acknowledgement to the participating women in the study and all participating midwives for their efforts concerning recruitment of study participants and practical realisation of the trial. We would like to thank José van Teylingen (research midwife) for her contributions to the trial conduction, Ria van der Bij (secretary of the Department of Obstetrics Academic Medical Center, Amsterdam) for her administra- 
tive support, Martine Eskes (obstetrician, MPH, Department of Obstetrics Academic Medical Center, Amsterdam) for her contributions to the steering committee of the trial and Ingeborg de Jonge (head Data Management of the Department of Clinical Epidemiology Leiden University Medical Center) for the database design and data entry.

\section{Funding}

This study was supported by ZONMw (The Netherlands Organisation for Health Research and Development), project number 22000036.

\section{References}

1 WHO. WHO: recommended definitions, terminology and format for statistical tables related to the perinatal period and use of a new certificate for cause of perinatal deaths. Acta Obstet Gynecol Scand 1977;56:247-53.

2 Hilder L, Costeloe K, Thilaganathan B. Prolonged pregnancy: evaluating gestation-specific risks of fetal and infant mortality. $\mathrm{Br} J$ Obstet Gynaecol 1998;105:169-73.

3 Bakketeig L, Bergsjø P. Post-term pregnancy: magnitude of the problem. In: Chalmers I, Enkin MW, Keirse MJNC, editors. Effective Care in Pregnancy and Childbirth. Oxford, UK: Oxford University Press; 1989. p. 765-75.

4 Munro KJ, Johnson RW, Philips MH. Historical Review of British Obstetrics and Gynaecology, 1800-1950. Edinburgh, UK: Livingstone, 1954.

5 Mitchell MD, Flint AP, Bibby J, Brunt J, Arnold JM, Anderson AB, et al. Rapid increases in plasma prostaglandin concentrations after vaginal examination and amniotomy. BMJ 1977;2:1183-5.

6 Keirse MJ, Thiery M, Parewijck W, Mitchell MD. Chronic stimulation of uterine prostaglandin synthesis during cervical ripening before the onset of labor. Prostaglandins 1983;25:671-82.

7 Boulvain M, Stan C, Jrion O. Membrane sweeping for induction of labour. Cochrane Database Syst. Rev. 2005 Jan 25;(1): CD 000451. Review.

8 Allott HA, Palmer CR. Sweeping the membranes: a valid procedure in stimulating the onset of labour? Br J Obstet Gynaecol 1993;100: 898-903.

9 Berghella V, Rogers RA, Lescale K. Stripping of membranes as a safe method to reduce prolonged pregnancies. Obstet Gynecol 1996;87: 927-31.

10 Cammu H, Haitsma V. Sweeping of the membranes at 39 weeks in nulliparous women: a randomised controlled trial. Br J Obstet Gynaecol 1998; 105:41-4.

11 McColgin SW, Hampton HL, McCaul JF, Howard PR, Andrew ME, Morrison JC. Stripping membranes at term: can it safely reduce the incidence of post-term pregnancies? Obstet Gynecol 1990;76: 678-80.

12 Crane J, Bennett K, Young D, Windrim R, Kravitz H. The effectiveness of sweeping membranes at term: a randomized trial. Obstet Gynecol 1997;89:586-90.

13 Dare FO, Oboro VO. The role of membrane stripping in prevention of post-term pregnancy: a randomised clinical trial in Ile-lfe, Nigeria. J Obstet Gynaecol 2002;22:283-6.

14 Doany W, McCarty J. Outpatient management of the uncomplicated postdate pregnancy with intravaginal prostaglandin E2 gel and membrane stripping. J Matern Fetal Med 1997;6:71-8.

15 el Torkey M, Grant JM. Sweeping of the membranes is an effective method of induction of labour in prolonged pregnancy: a report of a randomized trial. Br J Obstet Gynaecol 1992;99:455-8.

16 Goldenberg M, Dulitzky M, Feldman B, Zolti M, Bider D. Stretching of the cervix and stripping of the membranes at term: a random- ised controlled study. Eur J Obstet Gynecol Reprod Biol 1996;66: 129-32.

17 Gupta R, Vasishta K, Sawhney H, Ray P. Safety and efficacy of stripping of membranes at term. Int J Gynaecol Obstet 1998;60:115-21.

18 Magann EF, McNamara MF, Whitworth NS, Chauhan SP, Thorpe RA, Morrison JC. Can we decrease postdatism in women with an unfavorable cervix and a negative fetal fibronectin test result at term by serial membrane sweeping? Am J Obstet Gynecol 1998;179:890-94.

19 Magann EF, Chauhan SP, McNamara MF, Bass JD, Estes CM, Morrison $J C$. Membrane sweeping versus dinoprostone vaginal insert in the management of pregnancies beyond 41 weeks with an unfavorable cervix. J Perinatol 1999;19:88-91.

20 Salamalekis E, Vitoratos N, Kassanos D, Loghis C, Batalias L, Panayotopoulos $\mathrm{N}$, et al. Sweeping of the membranes versus uterine stimulation by oxytocin in nulliparous women. A randomized controlled trial. Gynecol Obstet Invest 2000;49:240-43.

21 Swann O. Induction of labor by stripping membranes. Obstet Gynecol 1958;11:74-8.

22 Tannirandorn Y, Jumrustanasan T. A comparative study of membrane stripping and nonstripping for induction of labor in uncomplicated term pregnancy. J Med Assoc Thai 1999;82:229-33.

23 Weissberg SM, Spellacy WN. Membrane stripping to induce labor. J Reprod Med 1977;19:125-7.

24 Wiriyasirivaj B, Vutyavanich T, Ruangsri RA. A randomized controlled trial of membrane stripping at term to promote labor. Obstet Gynecol 1996;87:767-70.

25 Wong SF, Hui SK, Choi H, Ho LC. Does sweeping of membranes beyond 40 weeks reduce the need for formal induction of labour? BJOG 2002;109:632-6.

26 Schulz KF, Grimes DA. Unequal group sizes in randomised trials: guarding against guessing. Lancet 2002;359:966-70.

27 Bishop EH. Pelvic scoring for elective induction. Obstet Gynecol 1964;24:266-8.

28 Bonsel GJ, van der Maas PJ. At the Cradle of the Future. Scenarios for Health Care Concerning Human Reproduction 1995-2010. STG Stichting Toekomstscenario's Gezondheidszorg. Houten/Diegem, The Netherlands: Bohn Stafleu Van Loghum, 1994.

29 Elferink-Stinkens PM, van Hemel OJ, Brand R, Merkus JM. The perinatal database of the Netherlands. Eur J Obstet Gynecol Reprod Biol 2001;94:125-38.

30 Boulvain M, Fraser WD, Marcoux S, Fontaine JY, Bazin S, Pinault JJ, et al. Does sweeping of the membranes reduce the need for formal induction of labour? A randomised controlled trial. Br J Obstet Gynaecol 1998; 105:34-40.

31 Harris BA Jr, Huddleston JF, Sutliff G, Perlis HW. The unfavorable cervix in prolonged pregnancy. Obstet Gynecol 1983;62:171-4.

32 Adair CE, Kowalsky L, Quon H, Ma D, Stoffman J, McGeer A, et al. Risk factors for early-onset group B streptococcal disease in neonates: a population-based case-control study. CMAJ 2003;169:198-203.

33 Lyytikainen O, Nuorti JP, Halmesmaki E, Carlson P, Uotila J, Vuento R, et al. Invasive group B streptococcal infections in Finland: a populationbased study. Emerg Infect Dis 2003;9:469-73.

34 Schuchat A Group B streptococcus. Lancet 1999;353:51-6.

35 Levine EM, Strom CM, Ghai V, Barton JJ. Intrapartum management relating to the risk of perinatal transmission of group B streptococcus. Infect Dis Obstet Gynecol 1998;6:25-9.

36 Lieu TA, Mohle-Boetani JC, Ray GT, Ackerson LM, Walton DL. Neonatal group B streptococcal infection in a managed care population. Perinatal Group B Streptococcal Infection Study Group. Obstet Gynecol 1998;92:21-27.

37 Schrag S, Gorwitz R, Fultz-Butts K, Schuchat A. Prevention of perinatal group $B$ streptococcal disease. Revised guidelines from CDC. MMWR Recomm Rep 2002;51:1-22. 